\title{
Mechanical, rheological, fatigue, and degradation behavior of PLLA, PGLA and PDGLA as materials for vascular implants
}

\author{
Magdalena Bartkowiak-Jowsa • \\ Romuald Będziński • Anna Kozłowska • \\ Jarosław Filipiak · Celina Pezowicz
}

Received: 22 March 2011 / Accepted: 8 October 2012 / Published online: 24 October 2012

(C) The Author(s) 2012. This article is published with open access at Springerlink.com

\begin{abstract}
The biggest challenge in the treatment of arterial stenosis remains the issue of optimization of stent design. Despite continuous improvement in surgical techniques and use of intensive pharmacotherapy, the results of stent coronary interventions may be unsatisfactory, and long-term interaction of a metal implant with a blood vessel results in complications such as recurrent stenosis and thrombosis. Therefore, it is necessary to search for new materials and stent designs to obtain a stent capable of restoring flow in the vessel and disappearing after fulfilling its function. Such stent must also be compatible with the vessel wall to enable regeneration of new structure of endothelium and deeper artery layers damaged during implantation. Consequently, there is ongoing search for functional solutions with minimum effects
\end{abstract}

M. Bartkowiak-Jowsa ( $\varangle)$ · J. Filipiak · C. Pezowicz Wrovasc - Integrated Cardiovascular Centre, Regional Specialist Hospital in Wroclaw, Research and Development Centre, ul. H.M Kamieńskiego 73a, Wroclaw, Poland e-mail: magdalena.bartkowiak@pwr.wroc.pl

M. Bartkowiak-Jowsa · R. Będziński · J. Filipiak ·

C. Pezowicz

Division of Biomedical Engineering and Biomechanics, Wroclaw University of Technology, ul. Łukasiewicza 7/9, 50-371 Wroclaw, Poland

\section{A. Kozłowska}

Institute of Environmental Engineering, Wroclaw University of Technology, Wybrzeże S. Wyspiańskiego 27, 50-370 Wroclaw, Poland of long-term implant-tissue interaction. In light of the above, the team investigated the possibility of using biodegradable polymers already mentioned in the literature as a construction material for vascular stent. The study used three polyhydroxyacids based on lactic acid and glycolic acid: poly(l-lactide), poly(lactideco-glycolide) and poly(d,l-lactide-co-glycolide). The research focused on assessing changes in mechanical, thermomechanical, rheological, and fatigue properties during the process of hydrolytic degradation. The analysis also covered the rate of release of degradation products. The results of the conducted tests indicate the possibility of developing a vascular stent with biodegradable polymers.

Keywords Biodegradable polymers $\cdot$ Mechanical properties · Rheological properties · DMA analysis . Hydrolytic degradation · Coronary stent

\section{Introduction}

One of the most commonly used methods of treatment of stenosis of blood vessels is stent angioplasty. The procedures involves introduction of a stent to the affected vessel, which after expansion "irons" the atherosclerotic plaque into the vessel wall, restores its patency, and supports it. The scaffolding function of a stent is most important during the first few weeks after surgery. Later on its presence is redundant and in many cases even dangerous. As a foreign body stent 
causes adverse effects such as restenosis or thrombosis, which lead to restenosis. Given the above, it can be concluded that an ideal stent should support vessel wall after angioplasty and biomechanically support vascular functions for the time needed for its regeneration, and after fulfilling that task it would degrade and disappear.

The first attempts to create biodegradable stent date back to the 1980s [1]. Over the years materials used were biodegradable polymers as well as biodegradable metals such as iron or magnesium. So far, several biodegradable stents were tested clinically. The first fully biodegradable stent implanted in humans was Igaki-Tamai stent made of poly(l-lactide) (PLLA). At the 10-year clinical follow up, stent showed impressive results. There were only 7 deaths during 10 years, whilst cumulative rates of target lesion revascularization (TLR) were $16 \%$ at 1 year, $18 \%$ at 5 years and $28 \%$ at 10 years [2,3]. Igaki-Tamai stent didn't cause changes in external elastic membrane area and was mostly degraded within 3 years. Unfortunately, stent expansion required bringing it to the temperature of approx. $70{ }^{\circ} \mathrm{C}$ which was supposed to be the burden to surrounding tissues. For low-risk patients these concerns were not substantiated but the stent is available in Europe only for peripheral use [2]. The AMS-1 (Biotronik, Berlin, Germany) was the first metallic, magnesium biodegradable stent. Clinical trial (PROGRESS AMS) revealed rapid degradation, high rate of TLR (45\%) and $42 \%$ reduction in the lumen area at 12 months [4]. These observations led to modifications in the design and drug release profile (AMS-2 and AMS-3 stents), new clinical program is currently continued. The REVA stent (Boston Scientific, Natick, MA) was resorbable and radiopaque stent made of tyrosine polycarbonate [5]. Innovative, slide-and-lock design was introduced, through it was possible to reduce polymer strains during implantation for $75 \%$ compared with standard deformable stent. Unfortunately, inflammation caused by REVA stent was higher compared to patients with bare metal stents (BMS). Redesigned, drug-eluting stent ReZolve showed good initial results in preclinical trials [2, 6], clinical trials are in progress. The BVS stent backbone was made of poly(l-lactide) (PLLA) core and poly D,L-lactide acid (PDLLA) coating, containing antiproliferative drug everolimus. In a porcine coronary artery, polymer degraded 24 months after implantation. In the ABSORB I Trial, 12 months after implantation restenosis rates were slightly higher in case of BVS $(22.9 \%)$ compared to BMS stents (19.3\%). The mean percent acute recoil was also higher for BVS stent $(6.85 \pm 6.96 \%$ vs. $4.27 \pm 7.08 \%)$ [7-10]. On the basis of these results it was concluded that BVS stents were characterized by weaker scaffolding properties than the Xience V metallic implants. Last year (2011) three-years randomized trial ABSORB II on the safety and efficacy of DES Xience Prime stent and a biodegradable polymer Absorb BVS stent began.

According to the literature presented, initial clinical results of biodegradable stents are promising. Well-known biodegradable materials such as PLLA has proven its safety in the cardiovascular system $[1-3,7]$. Nevertheless, there are still some issues that have to be concerned to develop biodegradable stent technology. Some of them are: obtaining appropriate strength of the stent without increasing stent strut dimensions, ensuring uniformity of deformations at the implant/tissue interface, matching degradation time with tissue regeneration time to prevent late lumen loss or finding method of implantation that wouldn't cause damage to arterial cells. Therefore, the search is continued for new construction materials that would meet the exacting requirements for design and functionality of vascular stents. Probably, such material could be developed through modifications of the well-known and widely used biodegradable polymers. However, it requires detailed knowledge of their properties and their changes at various stages of degradation. Based on reports in the literature [11-13] and own experiences [14], three materials which have previously showed their potential be used for the construction of vascular stents have been selected. Those are polyhydroxyacid polymers of different composition, available from Boehringer Ingelheim: poly(1lactide) (Resomer ${ }^{\circledR}$ L 210S, PLLA), poly(l-lactide-coglycolide) (Resomer ${ }^{\circledR}$ LG 857S, PGLA), and poly(d,1lactide-co-glycolide) (Resomer ${ }^{\circledR}$ RG 509S, PDGLA). The scope of presented research included: analysis of static and dynamic mechanical, thermomechanical, rheological, and fatigue properties. Intensity of release of degradation products and a change in the mass of the examined specimens were also assessed. Analysis of the state of knowledge indicates that detailed test results of degradation dynamics of those polymers in the context of their use as basic materials for the development of composites and blends for vascular stent are currently unavailable in the literature. The results of presented research would be detailed characteristics of four biodegradable polymers, which are used 
in vascular system. These data could also be the basis for modification of investigated materials, for instance by developing blends and composites in order to obtain a material with properties better suited to the development of vascular stent, and provide valuable information when designing other biodegradable implants.

\section{Materials and methods}

The tests was carried out on oar-shaped specimens with the $25 \mathrm{~mm}$ measurement basis, $5 \mathrm{~mm}$ width, and $2 \mathrm{~mm}$ thickness (EN ISO 527-1:1996, type 1BA) made by injection molding. The observation period for the specimens made of PLLA was 24 months, made of PGLA -7 months, and made of PDGLA-1 month. The observation time covered the period until total degradation of the materials. The process of hydrolytic degradation was carried out in distilled water at $\mathrm{pH} \approx 7$ and a temperature of $37 \pm 1^{\circ} \mathrm{C}$. Distilled water can be used as an incubation fluid at the stage of testing changes in mechanical properties during hydrolytic degradation and to assess its duration [1517]. The physical parameters of the examined polymers analyzed in this work were determined for various stages of degradation. Before the research began, it was assumed that the studied parameters would be analyzed for $1 / 3$ and $2 / 3$ of the degradation time of the material. However, due to the inability to accurately estimate the degradation time before its conclusion, ultimately the observation periods covered: 1 day, $28 \pm 5 \%$ and $63 \pm 5 \%$ of the time of total loss of the mechanical properties, making it impossible to conduct further research. The statistical significance of the obtained results was analyzed using nonparametric statistical analyses: the Mann-Whitney test (study of the differences in the mechanical properties between months of incubation) and the Spearman correlation (coefficients of correlations between changes in the mechanical properties of the specimens and $\mathrm{pH}$ changes of the environment during degradation).

\subsection{Static properties}

Static mechanical properties and their changes as a function of degradation time were determined on the basis of uniaxial tensile test. The loading system used was MTS Mini Bionix 858 fitted with hydraulic grips ensuring constant clamping force on the tested specimens, and strains measurements were taken with the use of videoextensometer ME 46-350 made by Messphysik. The strain rate was $0.16 / \mathrm{min}$. [18]. The obtained characteristics $\sigma=f(\varepsilon)$ were used to determine Young's in the linear region of the stress-strain curve, tensile strength $\sigma$ and deformability $\varepsilon$ (Figs. 1, $2,3)$.

\subsection{Resistance to cyclic loading}

Fatigue tests were carried out at uniaxial stress state, performing one-sided cyclic tension with a frequency of $f=5 \mathrm{~Hz}$. The index of asymmetry of the cycle was adopted at $R=\sigma_{\min } / \sigma_{\max }=0.5$ based on the average values of diastolic and systolic pressure in the human circulatory system. During the tests the value of maximum stress $\sigma_{\max }$ did not exceed $50 \%$ of the value of breaking stress [19]. The adopted limit was $10^{6}$ loading cycles. Tests were carried out on the loading system MTS Mini Bionix 858. During the tests the examined specimens remained in the environment ensuring a constant degree of hydration and a temperature of $37 \pm 1{ }^{\circ} \mathrm{C}$.

\subsection{Creep test}

A creep test was carried out to determine resistance of the tested materials to constant loading. The tested specimens were subjected to uniaxial tensile loading with a constant $15 \%$ of the force causing failure of non-incubated specimen. Constant loading was applied for a period of $2 \cdot 10^{4} \mathrm{~s}$. As in the case of the earlier specimen tests, these tests were carried out at the loading station MTS Mini Bionix 858 and the specimens were placed in an environment ensuring constant humidity and temperature. The analyzed parameters included: creep speed $v_{p}$ with which the strain increased during the last $10^{4} \mathrm{~s}$ of the test, the creep modulus $E_{t}$ (EN ISO 899-1:2005), and the maximum strain (Fig. 4).

\subsection{Dynamic temperature-dependent mechanical properties}

In order to determine the stability of the mechanical properties of the analyzed polymers as a function of temperature, tests were carried out of the dynamic thermomechanical properties (Fig. 5). The tests 

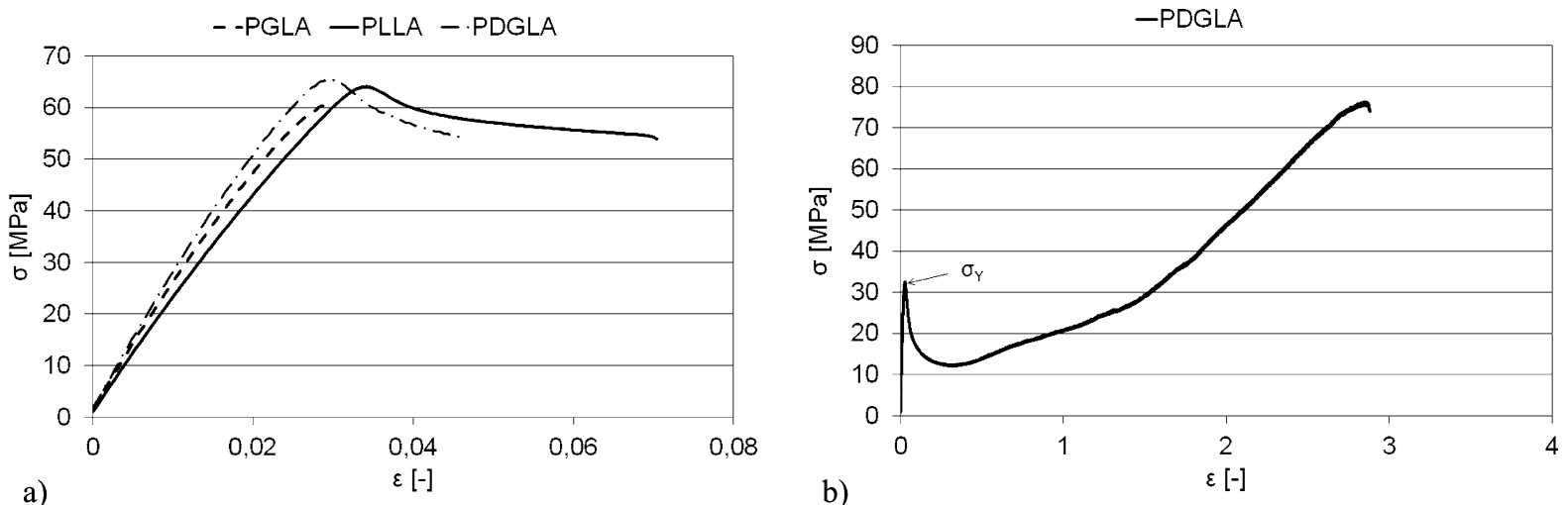

b)

Fig. 1 Stress-strain characteristics of: (a) PLLA, PGLA and PDGLA before incubation, (b) PDGLA after 1 day of incubation

were conducted on dynamic thermomechanical analyzer DMA 2980 made by TA Instruments. The tests were performed in the temperature range of -50 to $175^{\circ} \mathrm{C}$. Rectangular specimens measuring $36 \times 10 \times$ $4 \mathrm{~mm}$ were subjected to cyclic bending with a frequency of $1 \mathrm{~Hz}$ similar to the frequency of cardiac contractions; the bending amplitude was $25 \mu \mathrm{m}$.

\subsection{Changes in the $\mathrm{pH}$ of the incubation fluid}

Intense implant degradation promotes accumulation of the process product in the surrounding tissue, which may encourage development of a local inflammation [20]. Degradation products associated with hydrolysis of lactic and glycolic acids penetrating the incubation medium cause a change in $\mathrm{pH}$, namely acidification of the incubation environment. In many studies the $\mathrm{pH}$ value of the incubation environment is used as an indicator of the progress and intensity of the degradation process $[21,22]$. Also in the presented study the $\mathrm{pH}$ was measured to determine the degree of degradation of the analyzed polymers. $\mathrm{pH}$ measurements of the incubation environment for each specimen were performed weekly using $\mathrm{pH}$ meter CX 505 made by Elmetron with the resolving power of 0.001 (Fig. 6).

\section{Results}

3.1 Effect of hydration on the mechanical properties of materials

The study showed a significant effect of the process of hydrolytic degradation of the analyzed polymers on their basic mechanical parameters. Of interest is the effect of specimen hydration itself. Already 24-hour incubation results in significant changes of mechanical parameters of two of the analyzed polymers: PLLA $(E: p<0.01 \varepsilon: p<0.05)$ and PDGLA $\left(E, \sigma_{Y}, \varepsilon:\right.$ $\left.p<0.01 ; \sigma, \varepsilon_{Y}: p<0.05\right)$, values of PGLA mechanical properties changes not more than $10 \%$. In the case of PLLA and PGLA the character of $\sigma=f(\varepsilon)$ curves does not change. PDGLA specimens behave differently, as their $\sigma=f(\varepsilon)$ characteristic significantly changes its character (Fig. 1a, b). At the same time, values of all analyzed parameters change, for example hydrated specimens show an almost 100-fold increase in deformability compared to dry specimens. Due to large differences in the ranges of deformability of PDGLA and other materials, Fig. 1b only shows the $\sigma=f(\varepsilon)$ curve obtained for PDGLA to preserve readability the diagrams.

\subsection{Tests of static properties}

The rate of degradation is different for each of the examined polymers. Specifically, in the case of PLLA, following the five-month incubation period, decreases in the value of the Young's modulus (Fig. 2a) tensile strength $\sigma_{\max }$ (Fig. 2b), and deformability (Fig. 2c) amount to $7-15 \%$ compared to the initial value $(\sigma$ : $p<0.05$ ). After just 8 months these values increase enough to become equal or higher than the initial values. In the following months there is an increase in the Young's modulus and the stiffness of the material. After 15 months of incubation, these parameters are up to $60 \%$ higher than at the beginning of the degradation process $(\sigma, E, \varepsilon: p<0.05)$. This change is associated with faster degradation of the amorphous regions 

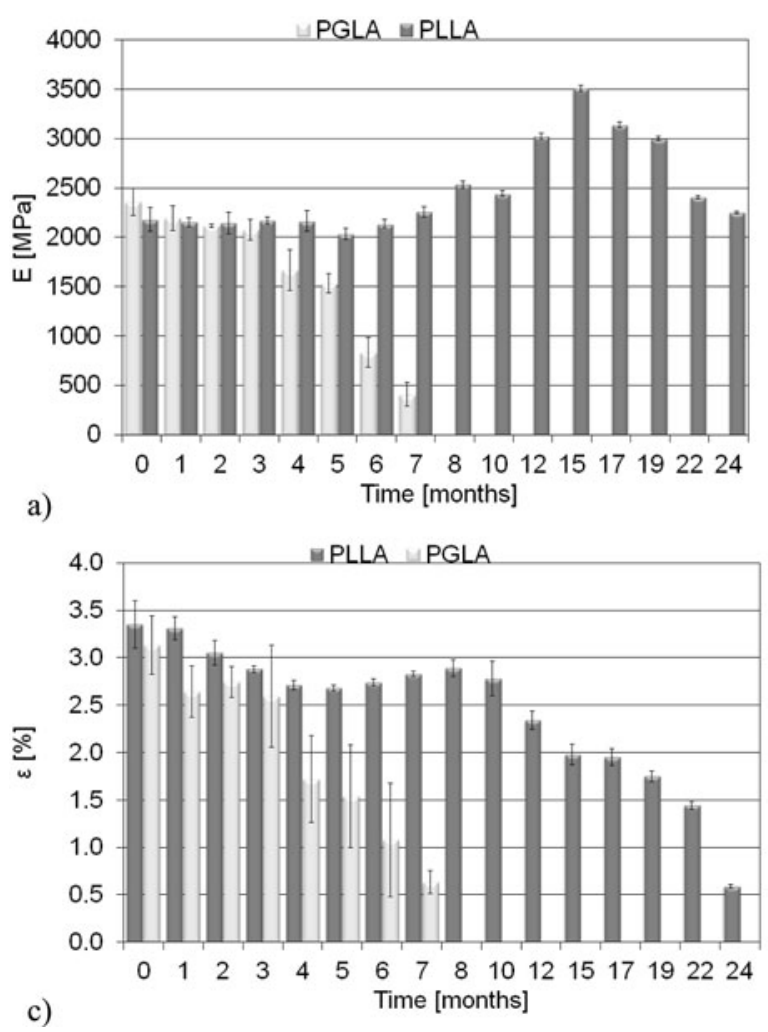

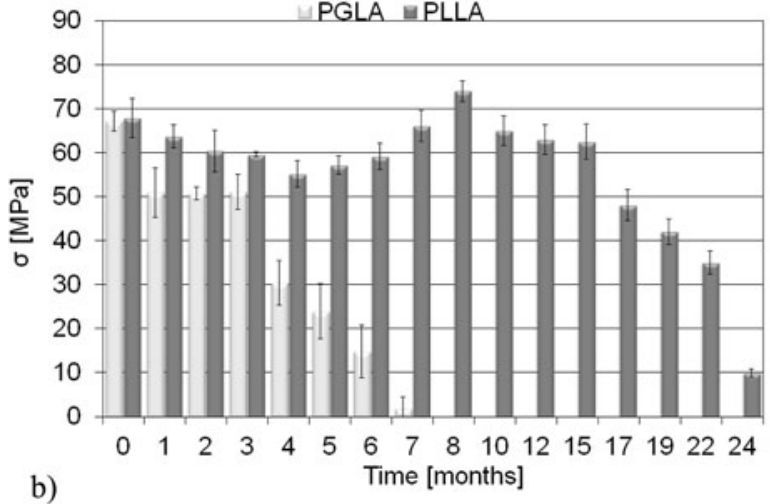

b)

Fig. 2 Changes in mechanical properties for PLLA and PGLA as a function of incubation time: (a) Young's modulus $E$, (b) tensile strength $\sigma,(\mathbf{c})$ strains corresponding to tensile strength $\varepsilon$

of the polymer [23, 24]. Moreover, degradation can be accompanied by the phenomenon of crystallization, i.e. recombination of monomers released due to degradation and formation of longer polymer chains. As a result, there is an improvement of the output mechanical properties of the material [25]. In subsequent months the mechanical properties of PLLA gradually decline until total physical degradation of the specimens prevents mechanical tests on the 25 th month of incubation.

In the case of PGLA the observed rate of change in the mechanical properties is definitively higher.

After 7 months of incubation there were significant $(p<0.01)$ decreases in the mechanical parameters of the specimens: the value of the Young's modulus dropped by about $80 \%$ (Fig. 2a), tensile strengthby $97 \%$ (Fig. 2b), and deformability—by about $80 \%$ (Fig. 2c). Some of the specimens could not be testes because of their physical degradation. The process of degradation is the fastest for PDGLA, where significant $(p<0.01)$ loss of mechanical properties pre- vented further testing in the fifth week of incubation. Already after 4 weeks of incubation the value of the Young's modulus (Fig. 3a) and yield stress $\sigma_{Y}$ (Fig. 3b) decreased by about $90 \%$ compared to the initial value.

\subsection{Tests of resistance to cyclic loading}

The process of hydrolytic degradation marks its impact on the ability to transfer cyclic loads. PLLA is able to transfer the limit number of cycles $10^{6}$ only after 1 day of incubation. In this case PGLA shows best results, as it is able to transfer the above number of cycles for all periods of observation. Due to the rate at which PDGLA loses its mechanical properties, no fatigue tests were conducted for that material.

\subsection{Creep test}

The degradation process is also visible in the case of rheological properties of the tested materials. The 

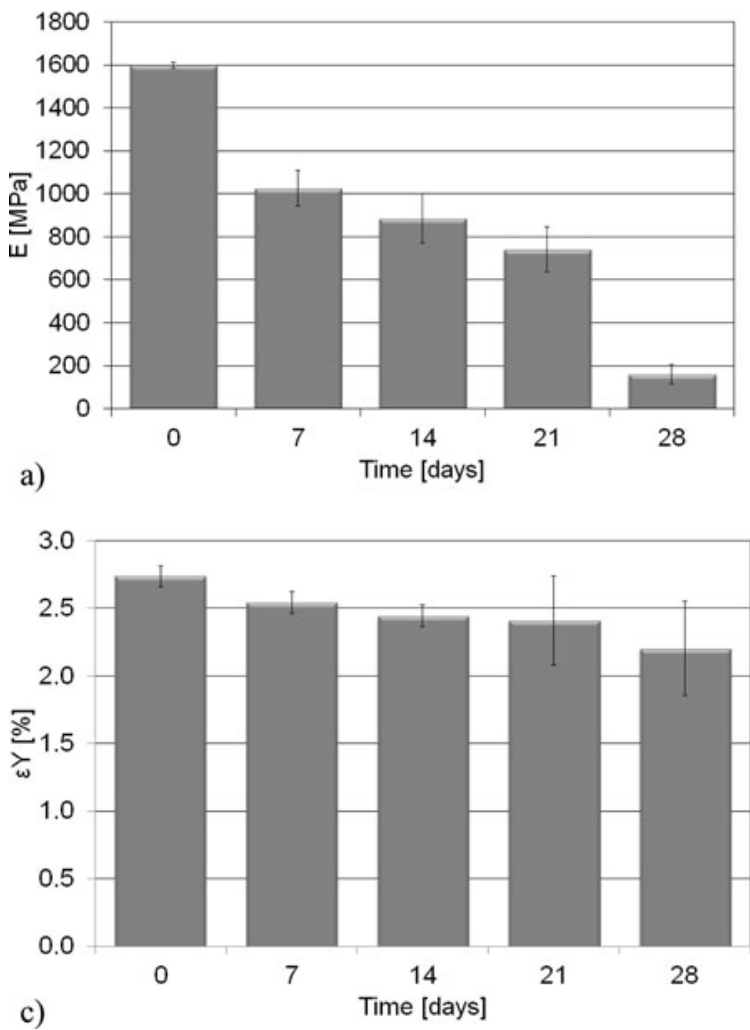
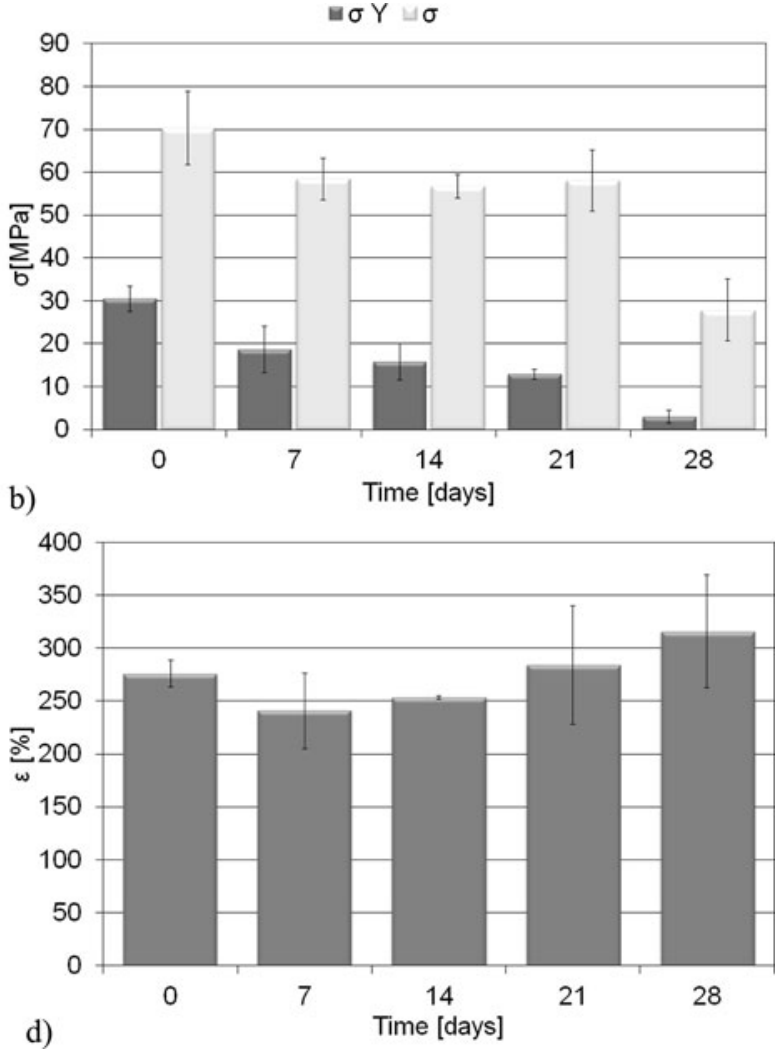

Fig. 3 Changes in the basic mechanical parameters for PDGLA as a function of incubation time: (a) Young's modulus $E$, (b) tensile strength $\sigma$, yield stress $\sigma_{Y}$ (see Fig. 1b), (c) yield strain, (d) strain corresponding to tensile strength

greatest, almost 7-fold increase in creep speed occurs in the case of PDGLA (Fig. 4c). For PLLA and PGLA that value stays at a similar level both after 1 day of incubation and during its duration (Fig. 4a-b). The maximum strain values after 1 day of incubation range from $0.5-3 \%$ for all materials. They change with the progress of degradation (Fig. 4a-c), and for PDGLA reach the maximum value of $11 \%$. In the case of PLLA the maximum strain value after 150 and 445 days of degradation is at a similar level as after 24hour incubation of specimens.

The phenomenon of creep strain decrease during degradation, as in the case of improvement of the strength properties of the material, is connected with the crystallization phenomenon taking place during degradation. New bonds formed between polymer chains during degradation restrict their mobility and susceptibility to creep [26].

\subsection{Dynamic temperature-dependent mechanical properties}

A desirable feature of the polymer intended for stent construction is its elasticity, which under conditions of dynamic loads is characterized by the storage modulus. At the body temperature of $37^{\circ} \mathrm{C}$ the values of that parameter for PLLA, PGLA, and PDGLA are, respectively: $2697 \mathrm{MPa}, 2414 \mathrm{MPa}$, and $2714 \mathrm{MPa}$. There is a sharp drop in the storage modulus in the temperature range of $40^{\circ} \mathrm{C}$ to $70^{\circ} \mathrm{C}$ in the case of PLLA and PGLA and $40^{\circ} \mathrm{C}$ to $60^{\circ} \mathrm{C}$ in the case of PDGLA. PLLA and PGLA have similar glass transition temperatures (respectively $68.0^{\circ} \mathrm{C}$ and $64.4^{\circ} \mathrm{C}$ ). PDGLA is characterized by the lowest glass transition temperature $\left(56.1^{\circ} \mathrm{C}\right)$, which is connected with degradation of the amorphous part and increased share of the crystalline phase an in the structure of the material (Fig. 5). 

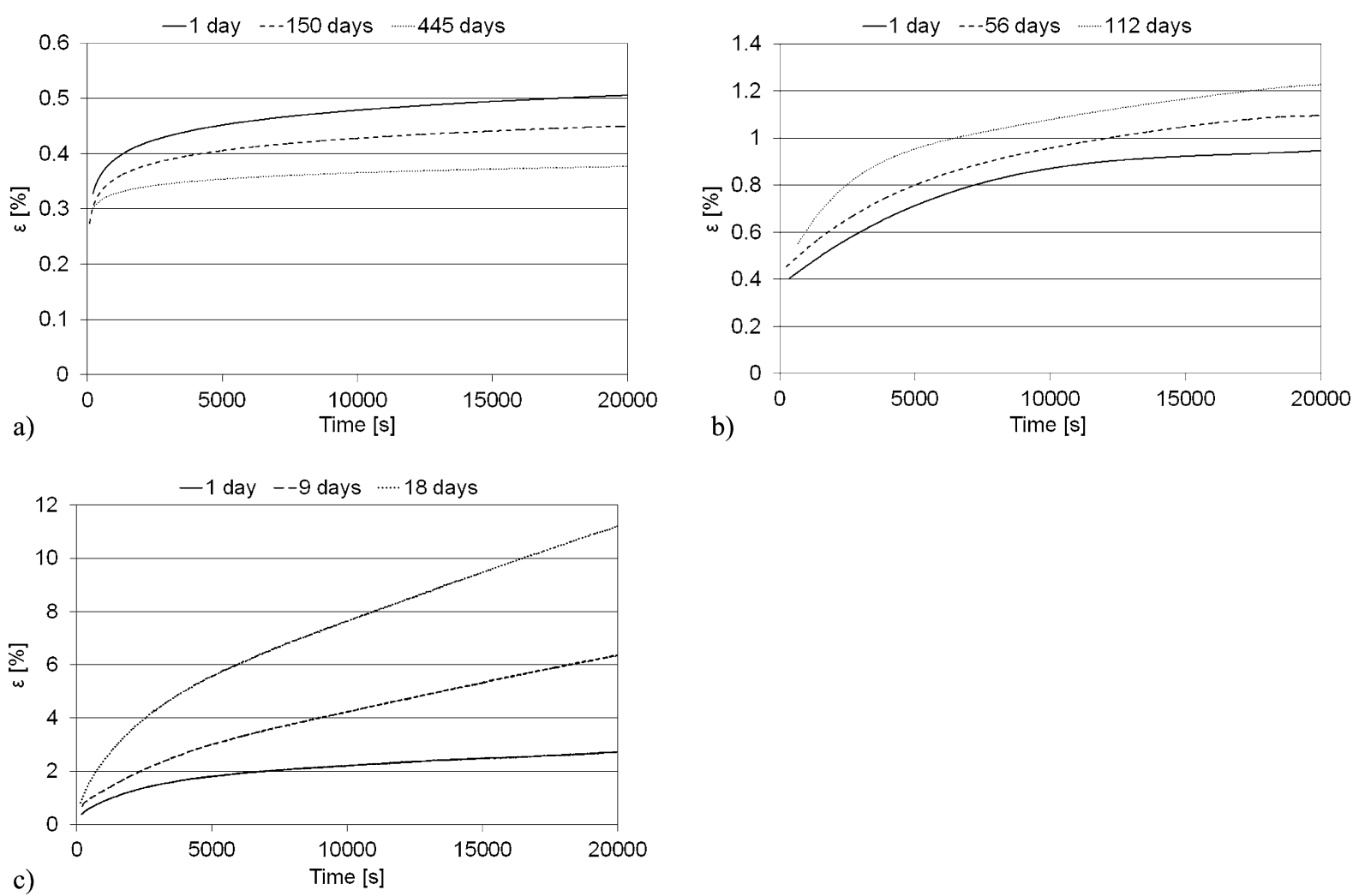

Fig. 4 Creep characteristics for the tested materials: (a) PLLA, (b) PGLA, (c) PDGLA

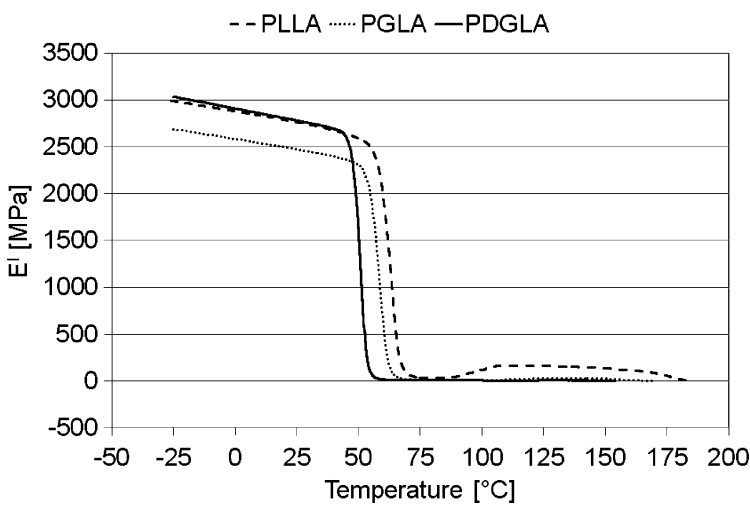

Fig. 5 The course of changes of the storage modulus $E^{I}$ as a function of temperature for PLLA, PGLA and PDGLA

\subsection{Concentration of degradation products}

The curves in Fig. 6 define the pH change of the tested polymers. The sawtooth character of the curves results from the need for period replacement of the incubation fluid during the experiment. The rate of $\mathrm{pH}$ change is slowest in the case of PLLA. Its value stays at a similar level throughout the incubation period (Fig. 6a) and can be approximated by a straight line with a slope of 0.017. In the case of PGLA there is an initial decrease in $\mathrm{pH}$ (Fig. 6b). In the case of PGLA the course of $\mathrm{pH}$ changes can be expressed by means of the regression function in the form of a straight line with a slope of 0.177 . The profile of $\mathrm{pH}$ changes is very different for PDGLA (Fig. 6c). In the initial period the $\mathrm{pH}$ change is small and the slope of the straight line describing that part of the characteristics reaches the value of 0.093 . After 3 weeks of incubation the $\mathrm{pH}$ value begins to decrease at a rate of 1.25 /week, which is reflected in the value of slope of the regression line at a level of 0.994.

The rapid decline in the $\mathrm{pH}$ value continues until the 8th week of incubation, when the $\mathrm{pH}$ value stabilizes at a level of about 1.5. At this stage the process of degradation is so advanced that if affects not only the structure of the material but also the geometry of the incubated specimen. 

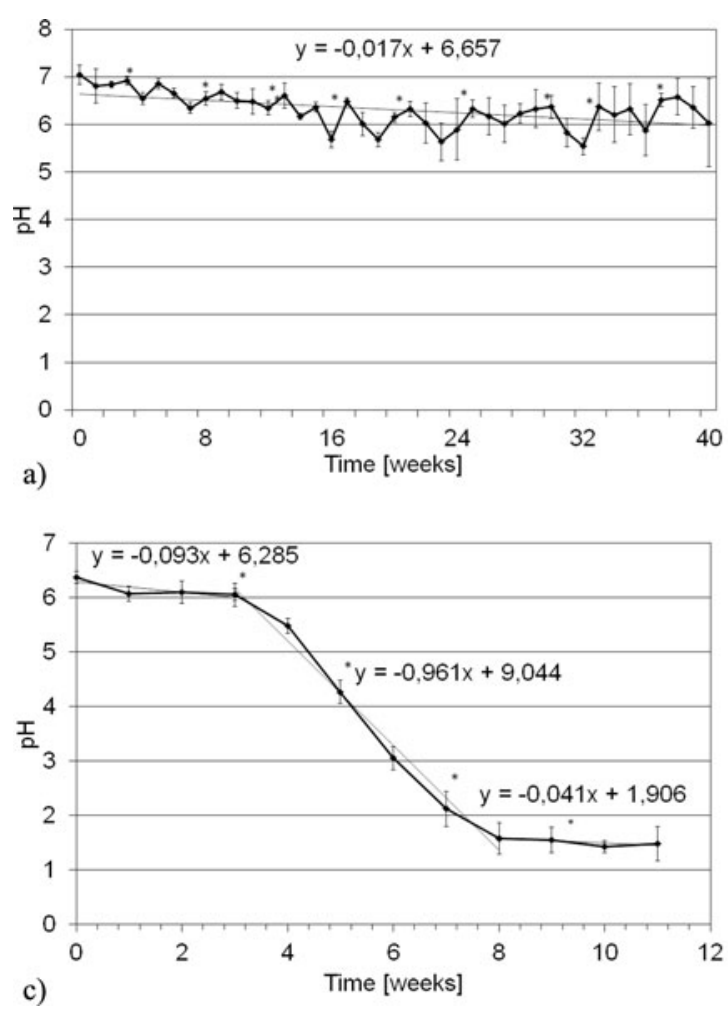

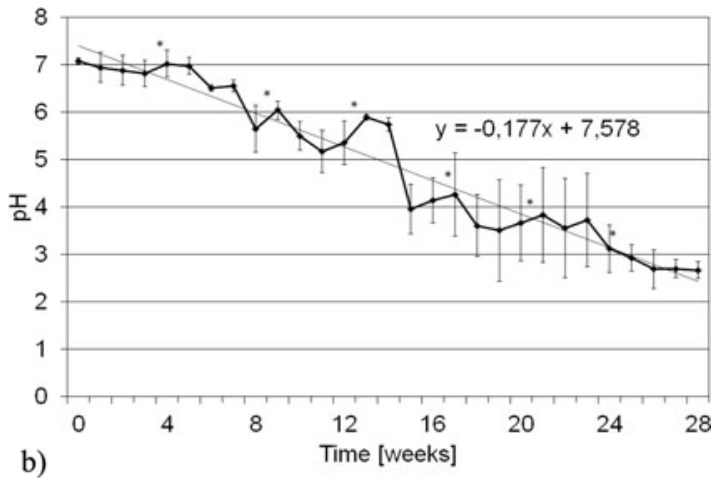

b)

Fig. 6 Changes in the pH value of the incubation solution: (a) PLLA, (b) PGLA, (c) PDGLA (*fluid replacement)

For each material there is a correlation (Spearman monotone correlation coefficient) between the Young's modulus, strength and deformability, and changes in the $\mathrm{pH}$ value in subsequent observation periods (Table 1). In the case of elastic modulus $(E)$ values, a negative correlation is due to an increase in the modulus connected with the process of crystallization during degradation causing an increase in this value at constant or slightly decreasing $\mathrm{pH}$ value.

\section{Discussion}

The obtained results indicate that mechanical properties of the analyzed materials change under the influence of aquatic environment. The degree of such changes depends on the content of the amorphous fraction, which, as revealed in the literature [27, 28], absorbs larger amounts of liquid. This was confirmed by the conducted studies where PDGLA degraded the fastest.

The results of research on degradation dynamics enabled selection of materials with degradation time
Table 1 Spearman coefficient of correlation between the strength parameters of the examined polymers and the $\mathrm{pH}$ values

\begin{tabular}{llrll}
\hline & & $E[\mathrm{MPa}]$ & $\sigma[\mathrm{MPa}]$ & $\varepsilon[\%]$ \\
\hline PLLA & $\mathrm{pH}$ & -0.427 & 0.628 & 0.824 \\
PGLA & $\mathrm{pH}$ & 0.913 & 0.965 & 0.971 \\
PDGLA & $\mathrm{pH}$ & 0.900 & 0.900 & 0.900 \\
\hline
\end{tabular}

best suited to the time of tissue regeneration. Taking into account the duration of the regeneration process of the individual layers of a vessel wall after their mechanical injury [29-31] e.g. as a result of angioplasty, we can conclude that the time needed for partial regeneration of the arterial wall is approx. 5-6 months. The period in which a biodegradable stent should act as a support should be similar. In the case of poly(l-lactide) (PLLA), following the five-month incubation period, the decrease in strength and deformability of the material does not exceed $10 \%$, which is favorable in terms of supporting vessel wall for the time needed for its regeneration. For poly(lactide-co-glycolide) (PGLA) a reduction in strength by $50 \%$ compared to the initial 
value takes place on the 4 moth of degradation, and after 6 months that material practically loses its ability to transfer any loads. Therefore, the PGLA degradation time is shorter than the expected period of regeneration of the vessel tissue, which means it cannot be used in pure form to build a stent to restore patency in vessels with advanced atherosclerotic lesions, whose fragmentation and compression into the vessel wall results in vascular damage and long regeneration lasting over 6 months. However, it can probably be used adjunctively as an implant after balloon angioplasty, preventing possible arterial dissection.

Poly(d,1-lactide-co-glycolide) (PDGLA) is characterized by the sharpest decline in the mechanical properties. For this material a $50 \%$ reduction in strength was recorded already in the 4th week of incubation, and the $E$ modulus value reaches a few percent of the initial value. Those are definitely unsatisfactory amounts from the viewpoint of the considered application of that material in vascular sent construction. However, it could be used e.g. as a drug-eluting outer layer of a polymer stent. On the other hand, it is interesting that the specimen maintains its mechanical continuity throughout the period and its deformability increases with the progression of the degradation process.

The obtained results for PDGLA were equally unfavorable in rheological tests. The examined polymer is susceptible to the creep phenomenon. On the 18th day of incubation the strains caused by the effect of constant force $F_{A}=0.15 F_{\max }$ over a time of $2 \cdot 10^{5} \mathrm{~s}$. amount to almost $12 \%$. In the same test the other polymers: PGLA and PLLA in the final stage of the incubation process (PGLA -112 days, PLLA—445 days) maximum strain values are, respectively, $1.23 \%$ and approx. $0.46 \%$.

The stability of the elastic properties of the material is important from the viewpoint of possible application of the tested polymers on implants used in the circulatory system. Tests of thermodynamic properties enabled assessment of the values of the storage modulus and determination of the range of temperatures in which analyzed materials demonstrate the desired elastic properties. With regard to the work of the implant, covering the temperature range of 35 to $42^{\circ} \mathrm{C}$, there was an approx. $2 \%$ observed decrease in the storage modulus. Glass transition temperatures are in the range of 56.1 to $68.0^{\circ} \mathrm{C}$ which indicates the stability of the structure of the tested materials in the useful tem- perature range, i.e. they will retain the glassy structure providing elastic properties of the material.

PLLA provides good mechanical properties but its degradation time is very long (24 months) compared to the time of tissue regeneration. One solution of this problem might be the application of an appropriate biodegradable filler, such as alginate fibers, which by enhancing liquid absorption at the phase boundary would be conducive to reducing the degradation time without compromising the mechanical properties of the material [32].

The question of the rate of degradation is connected with accumulation of degradation products in tissues surrounding the implant. Therefore, the rate of polymer degradation cannot be too fast not only because of the mechanical properties but also due to the biological effect of the released substances with the vessel tissue. Intense implant degradation, promoting accumulation of the degradation products in the tissue surrounding the implant, may encourage development of a local inflammation [20]. PDGLA is the material most intensely releasing degradation products; moreover, the right-handed variety of lactic acid that it releases (d-lactide) is poorly absorbed by human body [33] and its removal from the body takes much longer than in the case of the other forms of lactide. The rate of release of degradation products from PGLA and PLLA is less intense and left-handed variety of lactic acid (1-lactide), which is the main product of degradation of those polymers, is more readily absorbed by the body. This creates an opportunity to reduce the likelihood of inflammatory reactions.

\section{Summary}

In the literature there are many studies on the subject of degradation of polyhydroxyacids such as polylactide, poly(lactide-co-glycolide), or poly(d,l-lactide-coglycolide) [18]. Most of them focus on the analysis of chemical, structural, or thermal properties and only selected mechanical parameters of the examined polymers. Models are also formulated to describe progress of the processes taking place in the structure of the material during degradation $[17,19]$.

In this paper the authors are interested primarily in the effect of changes of chemical and structural parameters of a polymer subjected to degradation in the form of changes in the mechanical properties such as 
static and dynamic mechanical properties, rheological properties, or resistance to cyclic loading. Those information have practical significance when designing vascular stent or any other biodegradable implant. The obtained results are the basis for starting work towards development of polymer blends composites based on PLLA, PGLA or PDGLA to obtain materials better suited to fulfill the function of a construction material for vascular stents operating in the specific environment of the circulatory system. Therefore, the presented results constitute a reference point and form a basis for assessing properties of such optimized materials. The obtained results can also provide a basis for comparative analyses of biodegradable polymers synthesized in many laboratories and commercially available medical grade materials. They will also be used in the development of numerical models (using the method of finite elements) of stents at the construction design stage.

Acknowledgements This publication is part of project "Wrovasc-Integrated Cardiovascular Centre", co-financed by the European Regional Development Fund, within Innovative Economy Operational Program, 2007-2013 realized in Regional Specialist Hospital, Research and Development Centre in Wroclaw.

Open Access This article is distributed under the terms of the Creative Commons Attribution License which permits any use, distribution, and reproduction in any medium, provided the original author(s) and the source are credited.

\section{References}

1. Stack RE, Califf RM, Phillips HR et al. (1988) Interventional cardiac catheterization at duke medical center. Am J Cardiol 62:3F-24F

2. Onuma Y, Serruys PW (2011) Peripheral revascularization? bioresorbable scaffold: the advent of a new era in percutaneous coronary and peripheral revascularization? Circulation 123:779-797

3. Nishio S, Kosuga K, Igaki K, Okada M, Kyo E, Tsuji T et al. (2012) Long-term (> 10 years) clinical outcomes of first-in-man biodegradable poly-l-lactic acid coronary stents: Igaki-Tamai stents. Circulation 125(19):2343-2353

4. Erbel R, diMArio C, Bartunek J, Bonnier J, deBruyne B, Eberli FR et al. (2007) Temporary scaffolding of coronary arteries with bioabsorbable magnesium stents: a prospective non-randomised multicentre trial. Lancet 369(9576): 1839-1840

5. http://www.teamreva.com/company_news_RESORB.html

6. Grube E (2009) Bioabsorbable stent: the Boston Scientific and REVA technology. Presented at: EuroPCR; May 1922, 2009, Barcelona, Spain
7. Ormiston JA, Serruys PW, Regar E, Dudek D, Thuesen L, Webster MW et al. (2008) A bioabsorbable everolimuseluting coronary stent system for patients with single denovo coronary artery lesions (ABSORB): a prospective open-label trial. Lancet 371(15):899-907

8. Ormiston JA, Webster MW, Armstrong, G (2007) First-inHuman implantation of a fully bioabsorbable drug-eluting stent: the BVS poly-L-lactic acid everolimus-eluting coronary stent. Catheter Cardiovasc Interv 69:128-131

9. Serruys PW, Ormiston JA, Onuma Y, Regar E (2009) A bioabsorbable everolimus-eluting coronary stent system (ABSORB): 2-year outcomes and results from multiple imaging methods. Lancet 373:897-910

10. Domb AJ, Wiseman DM (1998) Handbook of biodegradable polymers. CRC Press, Boca Raton

11. Athanasiou KA, Agrawal CM, Barber FA, Burkhart SS (1998) Orthopaedic applications for PLA-PGA biodegradable polymers. Arthroscopy 7:726-737

12. Tamai H, Igaki K, Kyo E et al. (2000) Initial and 6-month results of biodegradable poly-l-lactic acid coronary stents in humans. Circulation 102:399-404

13. Serruys PW, Ormiston JA, Onuma Y, Regar E (2009) A bioabsorbable everolimus-eluting coronary stent system (ABSORB): 2-year outcomes and results from multiple imaging methods. Lancet 373:897-910

14. Bartkowiak-Jowsa M, Będziński R, Chłopek J, Filipiak J, Szaraniec B (2011) Comparative analysis of the deformation characteristics of biodegradable polymers considered as a material for vascular stents. Polymers 3:50-57

15. Gajowy J, Bednarz P, Laska J (2009) Degradation of polymer mixture of polyurethane and polylactide in simulated biological environment. Eng Biomater 89:213-216

16. Niu X, Luo Y, Li Y, Fu C, Chen J, Wang Y (2008) Design of bioinspired polymeric materials based on poly(D,L-lactic acid) modifications towards improving its cytocompatibility. J Biomed Mater Res 4:908-916

17. Sabino MA, Albuerne J, Müller AJ, Brisson J, Prud'homme RE (2004) Influence of in vitro hydrolytic degradation on the morphology and crystallization behavior of poly(pdioxanone). Biomacromolecules 2:358-370

18. Hashima K, Shotaro N, Inoue T (2010) Structure-properties of super-tough PLA alloy with excellent heat resistance. Polymer 17:3934-3939

19. El Fray M, Altstädt V (2003) Fatigue behaviour of multiblock thermoplastic elastomers. 2. Dynamic creep of poly(aliphatic/aromatic-ester) copolymers. Polymer 16:4643-4650

20. Hakkarainen M, Höglund A, Odelius K, Albertsson AC (2007) Tuning the release rate of acidic degradation products through macromolecular design of Caprolactone-based copolymers. J Am Chem Soc 129:6308-6312

21. Chłopek J, Morawska-Chochół A, Szaraniec B (2010) The influence of the environment on the degradation of polylactides and their composites. J Achiev Mater Manuf Eng 1:72-79

22. Szaraniec B, Ziąbka M, Morawska-Chochół A et al. (2007) In vitro hydrolytic degradation of resorbable fibres for medical implants applications. Eng Biomater 69-72:114-117

23. Joziasse CAP, Grijpma DW, Bergsma JE, Cordewener FW, Bos RRM, Pennings AJ (1998) The influence 
of morphology on the hydrolytic degradation of aspolymerized and hot-drawn poly(L-lactide). Colloid Polym Sci 276:968-975

24. Recum von H, Cleek RL, Eskin SG, Mikos AG (1995) Degradation of polydispersed poly(l-lactic acid) to modulate lactic acid release. Biomaterials 16:441-447

25. Han X, Pan J (2009) A model for simultaneous crystallisation and biodegradation of biodegradable polymers. Biomaterials 30:423-430

26. Tokiwa Y, Jarerat A (2004) Biodegradation of poly(1lactide). Biotechnol Lett 10:771-777

27. Weir NA, Buchanan FJ, Orr JF, Dickson GR (2004) Degradation of poly-L-lactide. Part 1: Physiological temperature degradation in vivo and in vitro. Proc Inst Mech Eng, H J Eng Med 218:307

28. Zhang X, Wyss UP, Pichora D, Goosen MFA (1992) An investigation of the synthesis and thermal stability of poly(DL-lactide). Polym Bull 27:623-629

29. Gennaro G, Ménard C, Michaud SE, Rivard A (2003) AgeDependent impairment of reendothelialization after arterial injury role of vascular endothelial growth factor. Circulation 107:230

30. Akimoto N, Miyazawa M, Torii T et al. (2008) Regeneration of the inferior vena cava with a bioabsorbable polymer implant: A histological study. J Sur Res 1:22-28

31. Walke W, Paszenda Z, Filipiak J (2005) Experimental and numerical biomechanical analysis of vascular stent. In: Achievements in mechanical \& materials engineering. AMME'2005. Proceedings of the 13th international scientific conference, Gliwice-Wisła, pp 699-702

32. Bartkowiak-Jowsa M, Kwiatkowska A, Szaraniec B, Chłopek J, Będziński R, Wikiewicz W (2011) Mechanical properties and degradation behaviour of PLA/alginate fiber composites. Eng Biomater 106-108:164-166

33. Gogolewski S (2003) Polymeric biomaterials. In: Biocybernetics and biomedical engineering 2000. Academic Publishing House Exit, Warsaw 